\title{
Inferior Hypogastric Block for the Treatment of Chronic Pelvic Pain
}

\author{
Ivan Urits (iD ${ }^{1,2}$, Ruben Schwartz ${ }^{3}$, Harish Bangalore Siddaiah ${ }^{2}$, Sathyadev Kikkeri ${ }^{2}$, David \\ Chernobylsky $^{4}$, Karina Charipova ${ }^{5}$, Jai Won Jung ${ }^{5}$, Farnad Imani (iD ${ }^{6}$, Mohsen Khorramian ${ }^{6, * *}$, \\ Giustino Varrassi (iD) ${ }^{7}$, Elyse M. Cornett ${ }^{2,}{ }^{*}$, Alan David Kaye ${ }^{2}$ and Omar Viswanath (iD ${ }^{2,8,9,10}$ \\ ${ }^{1}$ Southcoast Health, Southcoast Health Physicians Group Pain Medicine, Wareham, MA \\ ${ }^{2}$ LSU Health Shreveport, Department of Anesthesiology, Shreveport, LA \\ ${ }^{3}$ Mount Sinai Medical Center, Department of Anesthesiology, Miami Beach, FL \\ ${ }^{4}$ Cedars Sinai Medical Center, Los Angelos, CA \\ ${ }^{5}$ Georgetown University School of Medicine, Washington, DC \\ ${ }^{6}$ Pain Research Center, Department of Anesthesiology and Pain Medicine, Iran University of Medical Sciences, Tehran, Iran \\ ${ }^{7}$ Paolo Procacci Foundation, Via Tacito 7, Roma, Italy \\ ${ }^{8}$ University of Arizona College of Medicine-Phoenix, Phoenix, AZ \\ ${ }^{9}$ Creighton University School of Medicine, Department of Anesthesiology, Omaha, NE \\ ${ }^{10}$ Valley Anesthesiology and Pain Consultants - Envision Physician Services, Phoenix, AZ \\ "Corresponding author: LSU Health Shreveport, Department of Anesthesiology, Shreveport, LA. Email: ecorne@lsuhsc.edu \\ ${ }^{* *}$ Corresponding author: Pain Research Center, Department of Anesthesiology and Pain Medicine, Iran University of Medical Sciences, Tehran, Iran. Email: \\ mohsendoc51@gmail.com
}

Received 2020 December 16; Revised 2021 January 30; Accepted 2021 January 30.

\begin{abstract}
Context: Pelvic pain is described as pain originating from the visceral or somatic system localizing to the pelvis, the anterior abdominal wall at the level of or below the umbilicus, lumbosacral back in either men or women.

Evidence Acquisition: Narrative review.

Results: Chronic pelvic pain can be a complex disorder that may involve multiple systems such as urogynecological, gastrointestinal, neuromusculoskeletal, and psychosocial systems. The etiopathogenesis for chronic pain remains unknown for many patients. For achieving optimal patient management, a multimodal and individualized assessment of each patient is the best strategy. Conclusions: There are non-pharmacologic treatments as well as pharmacologic treatments. In addition to these treatment options, inferior hypogastric plexus block is a promising treatment modality.
\end{abstract}

Keywords: Chronic Pelvic Pain, Inferior Hypogastric Plexus, Trans-sacral Fluoroscopic Technique, Coccygeal Transverse Approach

\section{Context}

Pelvic pain is described as pain originating from the visceral or somatic system (T10 and below) localizing to the pelvis, the anterior abdominal wall at the level of or below the umbilicus, lumbosacral back, or the buttocks in either men or women (1-3). Men and women are affected by chronic pain syndromes related to interstitial cystitis, pelvic floor dysfunction, and chronic prostatitis (4).

Chronic Pelvic Pain in Women is described as persistent, noncyclic pain in the pelvic region severe enough to cause functional disability necessitating medical or surgical treatment and lasting more than three to six months with no definite identifiable disease process $(2,5,6)$. A thorough history and physical examination, including the abdominal and pelvic examination, are essential as it is mostly a diagnosis by exclusion (7). Chronic pelvic pain is multifactorial, and examples of pain conditions that might coexist are fibromyalgia, chronic fatigue syndrome, irritable bowel syndrome, temporomandibular joint disorders, and migraine headaches. Disorders commonly associated with it include endometriosis, adhesions, irritable

bowel syndrome, and interstitial cystitis (8). Pelvic congestion syndrome is one of the few factors which causes chronic pelvic pain in premenopausal women (9). A study conducted by Yosef et al. indicated the association of severity of chronic pelvic pain with abdominal wall pain, pelvic floor tenderness, painful bladder syndrome, a higher score on the pain catastrophizing scale, adult sexual assault, higher body mass index, current smoking, and family history of chronic pain (10).

Chronic pelvic pain is a potentially crippling complex disorder that frequently involves urogynecologic, gastrointestinal, neuromusculoskeletal, and psychosocial systems. This explains the wide range of symptoms, type, and 
severity that a patient might present to a clinician. Pain could be elicited on sitting, the sensation of a full bladder, urinating, defecating, ovulation, or ejaculation (11).

\subsection{Pelvic Cancer Pain}

Pelvic cancer can give rise to various pain types, including visceral, neuropathic, and somatic pain (12). Visceral pain (T10 - S5) is poorly localized, diffuse pain and can originate from abdominal structures, peritoneum, or omentum. Neuropathic pain results from damage to the nociceptive pathway anywhere from the peripheral or the central nervous system. The nervous system is susceptible to injury from tumor invasion, a complication of surgery, chemotherapy, or radiation therapy. Somatic pain (T12-S5) is a well localized, constant pain resulting from sensory signal input from skin, viscera, muscles, and joints (12).

\subsection{Epidemiology of Chronic Pelvic Pain}

Studies have elucidated the overall prevalence of chronic pelvic pain in women between $5.7 \%$ and $26.6 \%$, which translates to direct medical costs estimated at \$2.8 billion. Chronic Pelvic pain is associated with various subtypes $(3,13)$. Howard found that chronic pelvic pain was the reason for $10 \%$ of all referrals to gynecologists, $12 \%$ of all hysterectomies, and over $40 \%$ of gynecologic diagnostic laparoscopies. Endometriosis affects 5-10\% of women in their reproductive age. The etiopathogenesis for chronic pelvic pain remains unknown in 55 - 61 \% of women presenting with CPP, despite detailed diagnostic radiological tests and laparoscopy $(8,9,14)$.

A meta-analysis conducted by Van den Beuken-Van Everdingen et al. found that the prevalence of pain was 39.3\% after curative treatment, 55\% during anticancer treatment, and $66.4 \%$ in patients with advanced, metastatic, or terminal disease. $38 \%$ of the patients among the 122 studies reported moderate to severe pain (15). Chronic pain syndromes can be related to the effects of tumor invasion itself or anticancer therapy. Pelvic metastasis, hip joint syndrome, and malignant piriformis syndrome are related to the pelvis and hip. Others are Multifocal bone pain, Nociceptive pain syndrome due to bone metastasis, sacral syndrome, back pain. Visceral pain and Neuropathic pain syndromes result from the involvement of nerves at various levels such as nerve root (radiculopathy), plexus (plexopathy) (16). Treatment-related pain syndromes can occur due to hormonal therapy, radiation, chemotherapy, or surgery. Tumor related visceral pain can manifest as malignant perineal pain or ureteric obstruction. In addition to the neurologic syndromes, radiation therapy can cause lymphedema (16). Post-herpetic neuralgia manifesting as proctalgia fugax is very common following reduced immunity due to the cancer or its treatment
(12). Finally, the stress and anxiety of the disease can cause a psychophysiological pain syndrome (12).

Zondervan et al. conducted a cross-sectional analysis of the Primary Care database investigating 136 general practices in the UK. Interestingly, they discovered that the prevalence rates for chronic pelvic pain increased with age, but at the same time, the incidence decreased with age. Initially, they perceived that the rising prevalence in women older than 60 years could be due to the longer-lasting duration of pain in this subset of the population. But it was ultimately determined that the difference in prevalence could be due to the persistent regional disparities in incidence rather than the duration. This study concluded that the prevalence of chronic pelvic pain in primary care was comparable to migraine, back pain, and asthma, and possibly higher prevalence in the general population (17).

A population-based study by Grace and Zondervan in 2001 attempted to investigate the prevalence of chronic pelvic pain in women aged between 18 to 50 years. Among the 1,160 respondents to their questionnaire, the prevalence of chronic pelvic pain was $25.4 \%$. The use of health services by those who reported chronic pelvic pain was also investigated. Those consulting the health services belonged to women suffering from higher pain burden. $26.2 \%$ of women with chronic pelvic pain were diagnosed with irritable bowel syndrome, and $14.8 \%$ of women received endometriosis diagnosis (17).

\subsection{Pathophysiology of Chronic Pelvic Pain}

\subsubsection{Pelvic Cancer Pain}

Cancer pain can be debilitating as the tumor grows and invades the muscles, nerves, viscera, and surrounding structures, including bone. Nerve damage to the peripheral or central nervous system from the tumor or interventions presents with pain requiring opioid therapy or targeted interventions. The visceral disease results in distension or stretching of the capsule or compression and obstruction of viscera leading to pain, which might be associated with autonomic disturbances. Involvement of parietal peritoneum or omentum can result in pain. Activation of the nociceptors at the tissue level gives rise to somatic pain (12).

\subsubsection{Chronic Pelvic Pain}

The pelvic floor muscles comprised of piriformis, coccygeus, levator ani, internal anal sphincter, and perineal body provide support to the pelvic organs. The contraction of these muscles plays an important role in maintaining continence, while the abdominal pressure increases with forceful activity (3). The sacroiliac joint and pelvic ring provide stability to the pelvis. Pain in the pelvic area occurs 
with loss of stability and reduced load transferability between the trunk and lower extremity, and myofascial dysfunction of the pelvic floor(3). Problems originating either from the disc, joint, muscles, or nervous system can be important pain generator sources. Referred pain to the pelvis can occur from pathology in the nearby musculoskeletal system. Sympathetic innervation via hypogastric plexi from sacral and pelvic regions innervate the pelvic organs. These visceral afferents reach the upper lumbar and lower thoracic spinal cord similar to those visceral afferents from lower lumbar disc level-transmission of information from autonomic afferents projects as somatic symptoms. Nerve entrapment due to stretching, compression, fibrosis or suture, and injury due to prolonged second stage of labor, laser treatment to perineal structures could be an important source of origin of pelvic pain (3). Infection, trauma, or surgery can stimulate the production of bradykinin, leukotrienes, histamine, substance $\mathrm{P}, \mathrm{K}+, \mathrm{H}+$, which in turn activate the nerve endings and transmission of these impulses to the dorsal horn of the spinal cord lowers the pain threshold (18). Oxidative stress, neurogenic inflammation, and smooth muscle cell proliferation contribute to chronicity of pain $(6,19)$.

A case report by Rosenberg et al. described complete pain relief immediately following superior hypogastric plexus block in a patient with severe penile pain after transurethral resection of the prostate. The patient continued to be pain-free at 1, 2, 4, and 8 months follow-up (20).

The superior hypogastric plexus innervate the pelvic structures, including the bladder, urethra, perineum, prostate, penis, testis, descending colon, rectum, perineum, vulva, and internal genitalia except the ovary and fallopian tubes (21). The superior hypogastric plexus is retroperitoneal, located bilaterally at the lower third of the fifth lumbar vertebral body and upper third of the first sacral vertebral body proximity to the bifurcation of common iliac vessels. Axial computed tomography (CT) scanning aids in the visualization of vascular and soft tissue structures. Targeted superior hypogastric plexus blocks can be utilized for pelvic pain secondary to endometriosis. A disadvantage of this technique is intestinal perforation and radiation exposure (22). Wechsler et al. performed these blocks for patients with endometriosis and chronic pelvic pain using 20-gauge, $15 \mathrm{~cm}$ needles via the classic posterior approach in four patients and the anterior approach for the fifth patient. one patient experienced mild pain relief, three patients had considerable pain relief, the fifth patient had complete midline pain relief without any change in lateral pain and the sixth patient was totally pain-free. They abandoned the procedure on the seventh patient as the medication was accidentally injected into the peritoneal cavity. They concluded that CT-guided superior hypogastric block can be easily performed and could be used to assess whether chronic pelvic pain can be attenuated by blocking the superior hypogastric plexus (23).

\section{Evidence Acquisition}

Narrative Review. In 2020, we performed a comprehensive search for English-language studies related to inferior hypogastric block for the treatment of chronic pelvic pain. We searched the following databases: PubMed, Medline, SciHub, Cochrane database of systematic reviews, and Google scholar. We used the following combinations of keywords: Chronic pelvic pain; Inferior hypogastric plexus; Transsacral fluoroscopic technique; Coccygeal transverse approach Table 1 . We tried to include as many recent manuscripts as possible (within the last three years) but also included papers that were older than three years if they were particularly relevant to our topic. We also attempted to search for, use, and cite primary manuscripts whenever possible.

\section{Results}

3.1. Noninvasive Treatment Options for Chronic Pelvic Pain Syndrome

\subsubsection{Conservative}

For achieving optimal patient management, a multimodal and individualized assessment of each patient is the best strategy (24). Successful management of chronic pain conditions begins with a patient-centered approach and a conversation of realistic care goals. Patients must understand treatment options and their possible limitations and risks. This conversation is vital when addressing the symptoms of chronic pelvic pain. Non-pharmacologic treatment and more conservative options can begin with physical therapy as chronic pelvic pain is often associated with dysfunctional pelvic floor muscles (25). Patients might also benefit from osteopathic manipulative treatment (OMT) as it is used to address pelvic girdle biomechanics in gait and improve pain symptoms (26). A German pilot study found the use of OMT vs. physiotherapeutic exercise to improve quality of life and chronic pelvic pain syndrome symptom scores (27). Radial extracorporeal shockwave therapy is a non-pharmacologic treatment option and can improve quality of life and reduce pain domain in medication-refractory patients with urologic chronic pelvic pain syndrome (UCPPS) (28). The practice of yoga causes a reduction in pain symptoms and quality of life in patients with chronic pelvic pain and can be used 


\begin{tabular}{|c|c|c|c|}
\hline Study (y) & Groups Studied and Intervention & Results and Findings & Conclusions \\
\hline $\begin{array}{l}\text { Inferior hypogastric plexus block } \\
\text { affects sacral nerves and the } \\
\text { superior hypogastric plexus (2012) }\end{array}$ & Transsacral fluoroscopic technique & $\begin{array}{l}\text { Looked at five cadaveric studies to see } \\
\text { distribution of methylene blue dye } \\
\text { spread (blockage distribution) via } \\
\text { transsacral approach. }\end{array}$ & $\begin{array}{l}\text { Analgesia can be achieved through a } \\
\text { transsacral fluoroscopic technique for } \\
\text { inferior or superior hypogastric plexus } \\
\text { blockades. Altogether, the sympathetic } \\
\text { inferior hypogastric nerve blockage is } \\
\text { safe and effective for modulation of } \\
\text { pelvic visceral pain. }\end{array}$ \\
\hline $\begin{array}{l}\text { Inferior hypogastric plexus } \\
\text { blockade: A transsacral approach } \\
(2007)\end{array}$ & Transsacral fluoroscopic technique & $\begin{array}{l}\text { The technique for inferior hypogastric } \\
\text { plexus block via sacral foramen is } \\
\text { described with } 15 \text { blocks per } 11 \text { patients. }\end{array}$ & $\begin{array}{l}\text { With proper understanding of sacral } \\
\text { anatomy, expert needle steering } \\
\text { techniques, and fluoroscopy expertise, } \\
\text { this block can be performed safely and } \\
\text { effectively. }\end{array}$ \\
\hline $\begin{array}{l}\text { Inferior hypogastric plexus block } \\
\text { (2018) }\end{array}$ & Transsacral fluoroscopic technique & $\begin{array}{l}\text { Review of the two main approaches: } \\
\text { Transsacral and coccygeal. }\end{array}$ & $\begin{array}{l}\text { Despite the possibility of rectal } \\
\text { puncture, the newer coccygeal } \\
\text { approach has less disadvantages. }\end{array}$ \\
\hline $\begin{array}{l}\text { A new technique for inferior } \\
\text { hypogastric plexus block: A } \\
\text { coccygeal transverse approach-a } \\
\text { case report-(2012) }\end{array}$ & Coccygeal transverse approach & $\begin{array}{l}\text { Presentation of a case report where } \\
\text { coccygeal transverse approach was } \\
\text { performed. }\end{array}$ & $\begin{array}{l}\text { The new coccygeal transverse approach } \\
\text { allows for easier inferior hypogastric } \\
\text { plexus access and can be used for } \\
\text { diagnosis and treatment of lower } \\
\text { pelvic viscera. }\end{array}$ \\
\hline
\end{tabular}

as therapy along with conventional methods (29). Transcutaneous electrical nerve stimulations (TENS) is a safe and cost-effective treatment and can significantly improve pain scores in patients with idiopathic chronic pelvic pain. The impact of psychosocial and somatic factors on pain symptom severity in patients with CPPS is significant on a patient's physical and mental quality of life. Since higher depression levels correspond to lower physical and mental quality of life scores, integrated treatment consisting of psychological support and somatic aspects should be part of the mutli-modal approach to pain control for CPPS (30).

\subsubsection{Pharmacological Management}

Pain symptom management with pharmaceuticals is an essential component of the multimodal approach to treating CPPS. Common pharmaceutical treatments for CPPS include alpha-adrenergic blockers such as doxazosin, antibiotics, acetaminophen, gabapentinoids, and nonsteroidal anti-inflammatory drugs as they have demonstrated to offer symptomatic relief (31). Non-opioid analgesic drugs, including aspirin, acetaminophen, and NSAIDs, are limited to patients suffering from intractable pain from advanced cancer (32). Opioid analgesic medications are effective treatments for patients with intractable pain due to cancer (33). Most patients with chronic pain should receive oral administration of analgesic medication as the preferred route of administration. Alternative routes of administration exist for patients unable to receive oral medication (32). Intrathecal morphine is an effective treatment modality for intractable pain secondary to cancer of the pelvic organs (33). Continuous narcotic infusions utilizing a programmable portable pump is a safe and reliable narcotic delivery method for poorly controlled cancer pain in outpatients (34).

\subsection{Limitations of Treatment Options}

In a multimodal approach in the treatment of pain, each treatment option will have benefits and limitations. As such, a thorough evaluation of each patient is necessary to maximize outcomes with minimal side effects. Pharmacological management of CPPS is an essential component in treating pain symptoms, but each drug has their own individual side effect profile that must be taken into consideration. While the appropriate use of opioids is an important part of modern analgesic methods, no doubt overprescribing, misuse, and abuse of opioids has led to this crisis spiraling out of control (35). In 2016 and 2017, the number of drug overdose deaths due to opioids was $67 \%$ of all drug overdose deaths (36). Conditions resulting in chronic pain contribute to the risk of opioid use disorder (37). Opioidinduced dopamine release in the nucleus acumbens mediates reinforcing effects of opioid drugs contributing to misuse potential. A Pathological state of addiction is explained by an allostatic shift of the reward signaling pathway (38). A 3-point approach focused on prevention, treatment, and rehabilitation of patients currently afflicted or at future risk of opioid misuse and abuse (35). Careful adherence monitoring and building of the doctor-patient relationship can assist with identifying at-risk patients (35, 39).

Repeated risk stratification and state prescription drug monitoring programs, urine drug testing, pill counts, and behavioral analysis and assessments are highly utilized components of adherence monitoring. Although opioids 
might have great availability and multiple options, there is a lack of literature in managing chronic cancer pain with persistent opioid therapy. Clinical randomized controlled trials have shown no evidence supporting long-term opioid therapy for patients with chronic cancer pain. In contrast, there is an abundance of evidence of serious adverse complications related to long-term opioid therapy related to drug dosages, duration, and combination with benzodiazepines $(40,41)$. As wide recognition of the opioid epidemic is well established, an overhaul of previously established prevention strategies is greatly needed. In a health policy review manuscript, Manchikanti et al. describe a 3-tiered approach presented to Congress to create a new health policy designed to combat the opioid epidemic. The tiers consist of incorporating an aggressive education campaign of both physicians and the general public, facilitating easier access to non-opioid techniques and creating a national all schedules prescription electronic reporting act, and making buprenorphine more available for chronic pain $(42,43)$.

This manuscript does have limitations. The nature of a narrative review itself is subjective. Therefore, although we tried to remain unbiased in our inclusion of manuscripts to discuss in the paper (as evidenced by the Limitations section above) it is still possible that the authors were biased of which manuscripts to include (selection bias), the conclusions that were drawn from these studies, and how the studies were analyzed.

\subsection{Anatomy of the Inferior Hypogastric Block}

The inferior hypogastric plexus (IHP) exists as two plexuses, one in each of the right and left pelvic sidewalls (44). The IHP is a product of efferent sympathetic fibers arising from hypogastric and pelvic splanchnic nerves, preganglionic parasympathetic fibers from pelvic splanchnic nerves, and visceral afferent fibers from pelvic viscera. It is localized to the presacral tissue on either side of the rectum lying along the anterior surface of the sacrum while staying medial to the sacral foramina and ventral to the S2, S3, and S4 spinal segments (45). The IHP travels along the pelvic sidewall and is embedded in the lateral and presacral fascia at the level of the prostate, superficial to the prostatic fascia, and nearly inseparable from the prostatic vascular bundles as the fascial layer are shared and continuous (46). The anterior border or the IHP borders the prostate's posterior aspect at the level of the vesicoprostatic angle. The superior pole of the IHP resides at the level of the intersection of the vas deferens and ureter (47). Anatomic dissection studies of the IHP show the ureter to be the fundamental surgical point of reference representing the upper border of the IHP. This surgical point of reference is important when considering the etiology of chronic pelvic pain in women with a past surgical history of a hysterectomy, as resection of the IHP could have occurred intraoperatively (48).

$3 \mathrm{D}$ reconstruction of pelvic nerve elements using digitized histological and immunohistochemical transverse sections of a 16 and 17-week-old male fetus has allowed for precise location identification of the IHP and its nerve distribution. The 3D model demonstrated the majority of adrenergic fibers were mostly located in the superior portion of the IHP, and cholinergic fibers were primarily located in the inferior portion (49). Autonomic input to the penis is integrated with the IHP and communicated to the penis via cavernosal nerves. Risk stratification for treatments involving the IHP is crucial to avoid unintended consequences or side effects of treatments resulting in sexual dysfunction. Male patients report diminished orgasm intensity post radical prostatectomy due to loss of ejaculation (50). The IHP is challenging to identify and dissect because it shares vascular and fascial bundles with the seminal vesicles and prostate. These disadvantages confer a higher risk of damage to neurovascular bundles during radical prostatectomy leading to the development or worsening of CPPS and sexual dysfunction $(47,51)$.

In Interventional pain management, blockade of the hypogastric plexus can be a relatively common procedure. Commonly used Procedures for hypogastric plexus blockade previously focused on the blockade of the superior hypogastric plexus. Although superior hypogastric plexus blockade techniques have been refined using paravertebral or trans-discal approaches, the lower pelvic organs and genitalia are innervated by the inferior hypogastric plexus. They are not usually blocked as such with these methods. The trans-sacral approach, first introduced by Schultz in 2007, is a safe technique for diagnosing and treating chronic pain of the lower pelvic viscera (52).

\subsection{Indications for Inferior Hypogastric Block}

The autonomic nervous system's sympathetic limb contains some of the afferent and efferent neural fibers and pathways necessary for the creation or continuation of certain chronic pain conditions. Peripheral or other nerve injury combined with the loss of tonic sensory input damages information processing in the spinal cord resulting in inappropriate afferent sensory input leading to the increased efferent sympathetic stimulation. A sympathetic neural blockade may be a useful diagnostic tool in differentiating neuropathic pain processes between sympathetically mediated pain versus sympathetically independent pain (53). Although clinical trials provide statistical evidence supporting the use of sympathetic nerve blocks, a Cochrane review of these techniques recommended physi- 
cians take great caution and give much thoughtful consideration for incorporation into clinical practice (53).

Cancer pain is a complex pain state involving constant background pain, spontaneous pain, and motion-induced pain. Cancer pain is a mixed-mechanism pain state as it involves inflammatory, neuropathic, and cancer-specific pain. Bone is innervated by a dense network of sympathetic and sensory neurons. Alterations in bone homeostasis can sensitize the peripheral sensory and sympathetic nerves much more readily in cancer patients because of the density of sympathetic fibers in relation to a pathologic constant remodeling of the bone microenvironment (54). Neuropathic cancer pain (NCP) can be attributable to the cancer itself and caused by nerve damage attributable to chemotherapy, radiation therapy, and surgical therapy. Neuropathic cancer pain is further categorized as radiculopathy, plexopathy, peripheral neuropathies (55).

Pain management has improved significantly over the past fifty years. In 1954 the standard management of intractable cancer pain had two options, removal of the tumor or pain control with radiation and sex hormones. If those two options failed, physicians at the time could then attempt to treat the pain with narcotic analgesics, cobra venom, intravenous alcohol, and acetylsalicylic acid. Although modern treatment methods and protocols needed time to develop, even in 1954, it was known that narcotic medications have a substantially adverse side effect profile and alternative treatments such as analgesic blockade were superior to narcotic use (56).

Inferior hypogastric Plexus blockade is an effective and safe procedure for reducing pain symptoms of chronic pelvic pain. The block performed under fluoroscopic guidance using a trans-sacral approach and a local anesthetic/steroid combination to diagnose and treat sympathetically mediated chronic pain involving the lower pelvic viscera $(45,57)$. Acupuncture is a safe and effective method of treating pain symptoms in CPP. Inferior hypogastric plexus blockade is shown to be more effective at reducing pain symptoms than acupuncture and should be considered in a multimodal treatment approach to CPPS (58).

\subsection{Block Techniques}

There are several block techniques to modulate pain of the lower abdominal and pelvic viscera through the lower sympathetic chain's blockage. Several interventional options exist, including the trans sacral and coccygeal transverse approaches $(47,48,52)$.

\subsubsection{Trans sacral Fluoroscopic Technique}

Due to visceral pain transmission through the sympathetic nervous system, chronic pelvic pain manage- ment can be done via the ganglion impar and hypogastric plexus. Analgesia can be achieved through a trans sacral fluoroscopic technique for inferior or superior hypogastric plexus blockades. For the superior hypogastric plexus, there are multiple blockade techniques. Traditionally, the needle is inserted anteriorly to the L5 vertebral body via an oblique paravertebral direction. It is important to note that several studies report the L5 - S1 transdiscal approach is easier and safer (59).

The trans sacral approach is conducted using a 22gauge spinal needle. Fluoroscopy is used to both guided the needle through the posterior and anterior sacral foramen as well as confirm needle tip placement. The goal is to place the needle on the anterior sacral surface and inject local anesthetic for either diagnosis or therapy. The level of S2 dorsal foramen is usually selected due to easier visibility with fluoroscopy $(48,52,59)$.

It is important to note that when possible, it is recommended to perform the inferior hypogastric plexus block over the superior hypogastric plexus block due to safety concerns. Superior hypogastric plexus block placed the needle in close proximity to the bladder, bowel, and common iliac artery. The risk of injury to these structures is decreased with the inferior hypogastric plexus block. Nonetheless, due to its pre-sacral location, the transdical and paravertebral approaches are difficult to perform. Risks include damage to nerves, paraesthesia, hematomas, infection, vascular injury, and rectal puncture. Due to close proximity, the sacral spine can also be potentially damaged $(48,60,61)$.

In a recent study, five cadavers were injected with the inferior hypogastric plexus technique under trans sacral fluoroscopy. Anatomically, the 22-gauge spinal needle was inserted at S2, and needle position was confirmed with contrast Omniqaque dye solution. An injection of $10 \mathrm{~mL}$ methylene blue (diluted 1:50) was used to simulate the inferior hypogastric plexus blockage. After which, cadavers were dissected, and regional spread of dye was assessed. All five subjects showed that the blue dye distributed to the posterior rectum without diffusion to the lateral and anterior regions of the rectum $(47,61,62)$.

In all of the subjects, the S1 - S3 anterior sacral nerve roots were involved based on the spread of the dye. Diffusion was bilateral along the nerve roots, and all cadavers stained the L1 - S1 anterior surface of the superior hypogastric plexus. Overall, the trans sacral technique for inferior hypogastric block showed adequate distribution along the anterior sacral surface, dorsal rectal wall, without spreading to pelvic viscera. Specifically, the trans sacral approach promotes anesthetic distribution to superior hypogastric plexus and anterior sacral nerve roots (63).

Altogether, the sympathetic inferior hypogastric nerve 
blockage is safe and effective for modulation of pelvic visceral pain. In combination with other pain management modalities, the need for oral medications can be reduced (45).

\subsubsection{Coccygeal Transverse Approach}

The coccygeal transverse approach has been reported to have a decreased incidence of nerve damage, vascular injury, hematomas, infection, and rectal damage. While relatively new, the technique still has a risk of rectal puncture if the needle is advanced too far into the presacral area. Using lateral fluoroscopy, this can be prevented (59).

Performing this approach involves a 22-gauge bent needle with entry below the coccyx transverse process. Upon coccyx contact, the needle tip is moved medially and superiorly to the sacrococcygeal junction. The tip must reach the midway point of S2 and S3 junction. This reduces the risk of nerve damage, especially when paired with fluoroscopic imaging. Lateral fluoroscopic imaging is useful to find the sacrococcygeal area. When the needle is properly positioned, an aspiration test for stool or blood is performed. If negative, the contrast will cover the sacrococcygeal in a teardrop manner. The area will cover the retroperitoneal between the bowel and sacrococcygeal area. Afterwards, a mixture of $2 \%$ lidocaine $(2 \mathrm{~mL}), 0.25 \%$ bupivacaine $(2 \mathrm{~mL})$, and triamcinolone $(10 \mathrm{mg})$ can be injected (59, 64-68).

Patients report immediate pain relief with reduced supplementary pain control medication requirements. Also, as the new coccygeal transverse approach allows for easy inferior hypogastric plexus access with one needle puncture, it can be a useful alternative to the trans sacral technique. In essence, the coccygeal transverse approach to the inferior hypogastric plexus block is useful for diagnosing and treating chronic lower pelvic visceral pain (66, 68).

\subsubsection{Complications/Considerations}

The complications of these procedures involve infections, hematomas, nerve damage, paraesthesia, and vascular damage. In addition to the complications, the contraindications include pre-existing infection (local or systemic), coagulopathic disorders, distorted anatomy, and refusal by the patient (59).

\section{Conclusions}

Restriction of activities imposed by pain and a significant reduction in the function and quality of life can be very frustrating for patients. The prolonged suffering could manifest as marital discord, probable loss of employment, and have a tremendous impact on the consumption of health care resources and thereby economic burden (8). First of all, it can be a daunting task for the clinician to identify the specific pathological processes responsible for the origin of pain (3). In addition to early diagnosis and multimodal approach to pain relief, reassurance, elaborate discussion with the patient about care plans, and achievable treatment goals towards the effective restoration of function and cognitive behavioral therapy play a vital role in helping the patients learn handle the symptoms better (69). Advancements in the interventional procedures utilizing image guidance and involvement of dedicated pain specialist team focusing on the patient's individual needs are very helpful in providing treatment that is safe and efficacious. A patient-centered medical home managed by a primary care physician along with coordinated efforts from interdisciplinary team seems to be an ideal approach to provide optimum health care delivery for patients suffering from chronic pelvic pain (7). Future perspectives should include successful CPP evaluation and management needs a change in focus towards a biopsychosocial paradigm that targets not only individual peripheral pathology sources, but also the common underlying CPP core mechanisms. Using a multimodal, systemic approach, future research should further examine these core processes and how best to target them.

\section{Footnotes}

Authors' Contribution: Study concept and design, IU, RS, HBS, SK; Analysis and interpretation of data, DC, KC, JWJ, FI; Drafting of the manuscript, MK, GV, EMC, ADK, OV; Critical revision of the manuscript for important intellectual content, IU, RS, HBS, SK, DC, KC, JWJ, FI, MK, GV, EMC, ADK, OV; Statistical analysis, GV, EMC, ADK, OV.

\section{Conflict of Interests: None.}

Funding/Support: None.

\section{References}

1. Le PU, Fitzgerald CM. Pelvic pain: An overview. Phys Med Rehabil Clin NAm. 2017;28(3):449-54. doi:10.1016/j.pmr.2017.03.002. [PubMed: 28676357].

2. Howard FM. The role of laparoscopy in chronic pelvic pain: Promise and pitfalls. Obstet Gynecol Surv. 1993;48(6):357-87. doi: 10.1097/00006254-199306000-00001. [PubMed: 8327235].

3. Apte G, Nelson P, Brismée JM, Dedrick G, Justiz R, Sizer PS. Chronic female pelvic pain-part 1: Clinical pathoanatomy and examination of the pelvic region. Pain Pract. 2012;12(2):88-110. doi: 10.1111/j.15332500.2011.00465.x. [PubMed: 21615678]. 
4. Anderson RU, Wise D, Sawyer T, Nathanson BH, Nevin Smith J. Equal improvement in men and women in the treatment of urologic chronic pelvic pain syndrome using a multi-modal protocol with an internal myofascial trigger point wand. Appl Psychophysiol Biofeedback. 2016;41(2):215-24. doi: 10.1007/s10484-0159325-6. [PubMed: 26721470].

5. Speer LM, Mushkbar S, Erbele T. Chronic pelvic pain in women. Am Fam Physician. 2016;93(5):380-7. [PubMed: 26926975].

6. Adamian L, Urits I, Orhurhu V, Hoyt D, Driessen R, Freeman JA, et al. A comprehensive review of the diagnosis, treatment, and management of urologic chronic pelvic pain syndrome. Curr Pain Headache Rep. 2020;24(6):27. doi: 10.1007/s11916-020-00857-9. [PubMed: 32378039].

7. Bonnema R, McNamara M, Harsh J, Hopkins E. Primary care management of chronic pelvic pain in women. Cleve Clin JMed. 2018;85(3):21523. doi: 10.3949/ccjm.85a.16038. [PubMed: 29522389].

8. Howard FM. Chronic pelvic pain. Obstet Gynecol. 2003;101(3):594-611. doi: 10.1016/s0029-7844(02)02723-0. [PubMed: 12636968].

9. Borghi C, Dell'Atti L. Pelvic congestion syndrome: the current state of the literature. Arch Gynecol Obstet. 2016;293(2):291-301. doi: 10.1007/s00404-015-3895-7. [PubMed: 26404449].

10. Yosef A, Allaire C, Williams C, Ahmed AG, Al-Hussaini T, Abdellah MS, et al. Multifactorial contributors to the severity of chronic pelvic pain in women. Am J Obstet Gynecol. 2016;215(6):760 e1-760 e14. doi: 10.1016/j.ajog.2016.07.023. [PubMed: 27443813].

11. Hunter CW, Stovall B, Chen G, Carlson J, Levy R. Anatomy, pathophysiology and interventional therapies for chronic pelvic pain: a review. Pain Physician. 2018;21(2):147-67. [PubMed: 29565946].

12. Rigor BM. Pelvic cancer pain. J Surg Oncol. 2000;75(4):280-300. doi: 10.1002/1096-9098(200012)75:4<280::aid-jso13>3.0.co;2-q.

13. Ahangari A. Prevalence of chronic pelvic pain among women: An updated review. Pain physician. 2014;17(2):E141-7.

14. Zondervan KT, Becker CM, Koga K, Missmer SA, Taylor RN, Vigano P. Endometriosis. Nat Rev Dis Primers. 2018;4(1):9. doi: 10.1038/s41572-0180008-5. [PubMed: 30026507].

15. van den Beuken-van Everdingen MH, Hochstenbach LM, Joosten EA, Tjan-Heijnen VC, Janssen DJ. Update on prevalence of pain in patients with cancer: Systematic review and metaanalysis. J Pain Symptom Manage. 2016;51(6):1070-1090 e9. doi: 10.1016/j.jpainsymman.2015.12.340. [PubMed: 27112310].

16. Portenoy RK, Ahmed E. Cancer pain syndromes. Hematol Oncol Clin North Am. 2018;32(3):371-86. doi: 10.1016/j.hoc.2018.01.002. [PubMed: 29729775].

17. Grace VM, Zondervan KT. Chronic pelvic pain in New Zealand: prevalence, pain severity, diagnoses and use of the health services. Aust N Z J Public Health. 2004;28(4):369-75. doi: 10.1111/j.1467842x.2004.tb00446.x. [PubMed:15704703].

18. Ploteau S, Labat JJ, Riant T, Levesque A, Robert R, Nizard J. New concepts on functional chronic pelvic and perineal pain: Pathophysiology and multidisciplinary management. Discovery medicine. 2015;19(104):185-92.

19. Dellabella M, Milanese G, Sigala S, d'Anzeo G, Arrighi N, Bodei S, et al. The role of the prostatic stroma in chronic prostatitis/chronic pelvic pain syndrome. Inflamm Res. 2009;58(12):829-36. doi: 10.1007/s00011009-0086-7. [PubMed: 19757087].

20. Rosenberg SK, Tewari R, Boswell MV, Thompson GA, Seftel AD. Superior hypogastric plexus block successfully treats severe penile pain after transurethral resection of the prostate. Reg Anesth Pain Med.1998;23(6):618-20. doi: 10.1016/s1098-7339(98)90092-x. [PubMed: 9840861].

21. Tavakoli F, Yousefshahi F, Dabir A, Majedi H. Superior hypogastric plexus block in the lateral position: A case report. Anesth Pain Med. 2019;9(5). doi: 10.5812/aapm.94706. [PubMed: 31903333]. [PubMed Central: PMC6935289].

22. Nagpal AS, Moody EL. Interventional Management for Pelvic Pain. Phys Med Rehabil Clin $N$ Am. 2017;28(3):621-46. doi: 10.1016/j.pmr.2017.03.011. [PubMed: 28676368].
23. Wechsler RJ, Maurer PM, Halpern EJ, Frank ED. Superior hypogastric plexus block for chronic pelvic pain in the presence of endometriosis: CT techniques and results. Radiology.1995;196(1):103-6. doi: 10.1148/radiology.196.1.7784552. [PubMed: 7784552].

24. Nickel JC, Shoskes DA, Wagenlehner FME. Management of chronic prostatitis/chronic pelvic pain syndrome (CP/CPPS): The studies, the evidence, and the impact. World J Urol. 2013;31(4):747-53. doi 10.1007/s00345-013-1062-y. [PubMed: 23568442].

25. Berghmans B. Physiotherapy for pelvic pain and female sexual dysfunction: An untapped resource. Int Urogynecol J. 2018;29(5):631-8. doi: 10.1007/s00192-017-3536-8. [PubMed: 29318334]. [PubMed Central: PMC5913379].

26. Moloney S, Talsma J, Pierce-Talsma S. Osteopathic manipulative medicine considerations in pelvic pain. J Am Osteopath Assoc. 2019;119(11):e42-3. doi:10.7556/jaoa.2019.130. [PubMed:31657833].

27. Marx S. [Chronic pelvic pain syndrome : Treatment options using osteopathy]. Urologe A. 2017;56(8):1008-16. doi:10.1007/s00120-017-03882. [PubMed: 28401276].

28. Salama AB, Abouelnaga WA. Effect of radial shock wave on chronic pelvic pain syndrome/chronic prostatitis. $J$ Phys Ther Sci. 2018;30(9):1145-9. doi: 10.1589/jpts.30.1145. [PubMed: 30214114]. [PubMed Central: PMC6127492].

29. Saxena R, Gupta M, Shankar N, Jain S, Saxena A. Effects of yogic intervention on pain scores and quality of life in females with chronic pelvic pain. Int J Yoga. 2017;10(1):9-15. doi: 10.4103/0973-6131.186155. [PubMed: 28149062]. [PubMed Central: PMC5225749].

30. Piontek K, Ketels G, Albrecht R, Schnurr U, Dybowski C, Brünahl CA, et al. Somatic and psychosocial determinants of symptom severity and quality of life in male and female patients with chronic pelvic pain syndrome. J Psychosom Res. 2019;120:1-7. doi: 10.1016/j.jpsychores.2019.02.010. [PubMed: 30929698].

31. Qin Z, Wu J, Tian J, Zhou J, Liu Y, Liu Z. Network Meta-Analysis of the Efficacy of Acupuncture, Alpha-blockers and Antibiotics on Chronic Prostatitis/Chronic Pelvic Pain Syndrome. Sci Rep. 2016;6:35737. doi: 10.1038/srep35737. [PubMed: 27759111]. [PubMed Central: PMC5069632].

32. Levy MH. Pharmacologic treatment of cancer pain. N Engl J Med. 1996;335(15):1124-32. doi: 10.1056/NEJM199610103351507. [PubMed: 8813044].

33. Wang JK. Intrathecal morphine for intractable pain secondary to cancer of pelvic organs. Pain. 1985;21(1):99-102. doi: 10.1016/03043959(85)90081-8.

34. Kerr IG, Sone M, DeAngelis C, Iscoe N, MacKenzie R, Schueller T. Continuous narcotic infusion with patient-controlled analgesia for chronic cancer pain in outpatients. Ann Intern Med. 1988;108(4):5547. doi: 10.7326/0003-4819-108-4-554. [PubMed: 2894791].

35. Stoicea N, Costa A, Periel L, Uribe A, Weaver T, Bergese SD. Current perspectives on the opioid crisis in the US healthcare system: A comprehensive literature review. Medicine (Baltimore). 2019;98(20). e15425. doi: 10.1097/MD.0000000000015425. [PubMed: 31096439]. [PubMed Central: PMC6531094].

36. Centers for Disease Control and Prevention. Annual Surveillance Report ofDrug-Related Risks and Outcomes-United States Surveillance Special Report.U.S:Centers for Disease Control and Prevention, U.S. Department of Health and Human Services; 2019.

37. Malik KM, Imani F, Beckerly R, Chovatiya R. Risk of opioid use disorder from exposure to opioids in the perioperative period: A systematic review. Anesth Pain Med.2020;10(1). e101339. doi: 10.5812/aapm.101339. [PubMed: 32337175]. [PubMed Central: PMC7158240].

38. Koob GF, Le Moal M. Neurobiological mechanisms for opponent motivational processes in addiction. Philos Trans $R$ Soc Lond B Biol Sci. 2008;363(1507):3113-23. doi: 10.1098/rstb.2008.0094. [PubMed: 18653439]. [PubMed Central: PMC2607326].

39. Blanco C, Wall MM, Okuda M, Wang S, Iza M, Olfson M. Pain as a Predictor of Opioid Use Disorder in a Nationally Representative Sample. Am 
J Psychiatry. 2016;173(12):1189-95. doi: 10.1176/appi.ajp.2016.15091179. [PubMed: 27444794].

40. Manchikanti L, Manchikanti KN, Kaye AD, Kaye AM, Hirsch JA. Challenges and concerns of persistent opioid use in cancer patients. Expert Rev Anticancer Ther. 2018;18(7):705-18. doi: 10.1080/14737140.2018.1474103. [PubMed: 29739242].

41. Manchikanti L, Kaye AM, Knezevic NN, McAnally H, Trescot AM, Blank $S$, et al. Responsible, safe, and effective prescription of opioids for chronic non-cancer pain: American Society of Interventional Pain Physicians (ASIPP) guidelines. Pain physician. 2017;20(2):S3-S92.

42. Manchikanti L, Sanapati J, Benyamin RM, Atluri S, Kaye AD, Hirsch JA. Reframing the prevention strategies of the opioid crisis: Focusing on prescription opioids, fentanyl, and heroin epidemic. Pain physician. 2018;21(4):309-26.

43. Hemati K, Zaman B, Hassani V, Imani F, Dariaie P. Efficacy of fentanyl transdermal patch in the treatment of chronic soft tissue cancer pain. Anesth Pain Med.2015;5(1). e22900. doi:10.5812/aapm.22900. [PubMed: 25789240]. [PubMed Central: PMC4350185].

44. Rogers Jr RM. Basic neuroanatomy for understanding pelvic pain. Am Assoc Gynecol Laparosc. 1999;6(1):17-25.

45. Mohamed SA, Ahmed DG, Mohamad MF. Chemical neurolysis of the inferior hypogastric plexus for the treatment of cancerrelated pelvic and perineal pain. Pain Res Manag. 2013;18(5):249-52. doi: 10.1155/2013/196561. [PubMed: 23717828]. [PubMed Central: PMC3805347]

46. Patel U. The prostate and seminal vesicles. In: Allan PL, Baxter GM, Weston MJ, editors. Clinical Ultrasound. 1.3 ed. Amsterdam, Netherlands: Elsevier Ltd; 2011. p. 572-92. doi: 10.1016/b978-0-7020-3131-1.00030-4.

47. Mauroy B, Demondion X, Drizenko A, Goullet E, Bonnal JL, Biserte J, et al. The inferior hypogastric plexus (pelvic plexus): Its importance in neural preservation techniques. Surg Radiol Anat. 2003;25(1):6-15. doi: 10.1007/s00276-002-0083-9. [PubMed: 12690518].

48. Mauroy B, Demondion X, Bizet B, Claret A, Mestdagh P, Hurt C. The female inferior hypogastric (= pelvic) plexus: Anatomical and radiological description of the plexus and its afferences-applications to pelvic surgery. Surg Radiol Anat. 2007;29(1):55-66. doi: 10.1007/s00276-0060171-3. [PubMed: 17186314].

49. Alsaid B, Bessede T, Karam I, Abd-Alsamad I, Uhl JF, Benoît G, et al. Coexistence of adrenergic and cholinergic nerves in the inferior hypogastric plexus: Anatomical and immunohistochemical study with 3D reconstruction in human male fetus. J Anat. 2009;214(5):645-54. doi: 10.1111/j.1469-7580.2009.01071.x. [PubMed: 19438760]. [PubMed Central: PMC2707089].

50. Bhasin S, Basson R. Sexual dysfunction in men and women. Williams textbook of endocrinology. Elsevier Saunders, Philadelphia, PA; 2011. p. 793-5.

51. Yousefshahi F, Tahmasebi M. Long-Lasting orthostatic hypotension and constipation after celiac plexus block; A case report. Anesth Pain Med. 2018;8(1). e63221. doi: 10.5812/aapm.63221. [PubMed: 29868459]. [PubMed Central: PMC5970361].

52. Schultz DM. Inferior hypogastric plexus blockade: A transsacral approach. Pain Physician. 2007;10(6):757.

53. Petersohn JD. Sympathetic neural blockade. Pain Procedures in Clinical Practice. Elsevier; 2011. p. 507-19.
54. Falk S, Bannister K, Dickenson AH. Cancer pain physiology. Br J Pain. 2014;8(4):154-62. doi: 10.1177/2049463714545136. [PubMed: 26516549]. [PubMed Central: PMC4616725].

55. Yoon SY, Oh J. Neuropathic cancer pain: Prevalence, pathophysiology, and management. Korean J Intern Med. 2018;33(6):1058-69. doi: 10.3904/kjim.2018.162. [PubMed: 29929349]. [PubMed Central: PMC6234399].

56. Bonica JJ. The management of pain of malignant disease with nerve blocks. Anesthesiology. 1954;15(2):134-5. contd. [PubMed: 13138931].

57. Amin MM, Ait-Allah AS, Ali Ael S, Salem RA, Ahmed SR, Alsammani MA. Inferior hypogastric plexus blockade versus acupuncture for the management of idiopathic chronic pelvic pain: A randomized clinical trial. Biomed J. 2015;38(4):317-22. doi: 10.4103/2319-4170.151034. [PubMed: 25673173].

58. Lee SH, Lee BC. Use of acupuncture as a treatment method for chronic prostatitis/chronic pelvic pain syndromes. Curr Urol Rep. 2011;12(4):288-96. doi: 10.1007/s11934-011-0186-0. [PubMed: 21472420].

59. Choi HS, Kim YH, Han JW, Moon DE. A new technique for inferior hypogastric plexus block: A coccygeal transverse approach, a case report. Korean J Pain. 2012;25(1):38-42. doi: 10.3344/kjp.2012.25.1.38. [PubMed: 22259715]. [PubMed Central: PMC3259136].

60. Campbell F, Collett BJ. Chronic pelvic pain. BrJAnaesth.1994;73(5):5713. doi: 10.1093/bja/73.5.571. [PubMed: 7826779].

61. Zondervan KT, Yudkin PL, Vessey MP, Dawes MG, Barlow DH, Kennedy $\mathrm{SH}$. Prevalence and incidence of chronic pelvic pain in primary care: evidence from a national general practice database. Br J Obstet Gynaecol. 1999;106(11):1149-55. doi: 10.1111/j.1471-0528.1999.tb08140.x. [PubMed: 10549959].

62. Vincent K. Chronic pelvic pain in women. Postgrad Med J. 2009;85(999):24-9. doi: 10.1136/pgmj.2008.073494. [PubMed: 19240284].

63. Turker G, Basagan-Mogol E, Gurbet A, Ozturk C, Uckunkaya N, Sahin S. A new technique for superior hypogastric plexus block: the posteromedian transdiscal approach. Tohoku J Exp Med. 2005;206(3):277-81. doi: 10.1620/tjem.206.277. [PubMed: 15942158].

64. Bosscher H. Blockade of the superior hypogastric plexus block for visceral pelvic pain. Pain Pract. 2001;1(2):162-70. doi: 10.1046/j.15332500.2001.01017.x. [PubMed: 17129292].

65. Waldman SD, Wilson WL, Kreps RD. Superior hypogastric plexus block using a single needle and computed tomography guidance: Description of a modified technique. Reg Anesth. 1991;16(5):286-7.

66. Kanazi G, Perkins F, Thakur R, Dotson E. New technique for superior hypogastric plexus block. Reg Anesth Pain Med.1999;24(5):473-6.

67. Erdine S, Yucel A, Celik M, Talu GK. Transdiscal approach for hypogastric plexus block. Reg Anesth Pain Med. 2003;28(4):304-8. doi: 10.1016/s1098-7339(03)00191-3. [PubMed: 12945023].

68. Nabil D, Eissa AA. Evaluation of posteromedial transdiscal superior hypogastric block after failure of the classic approach. Clin J Pain. 2010;26(8):694-7. doi: 10.1097|AJP.ob013e3181dedfa9. [PubMed: 20664339].

69. Imani F, Varrassi G. Ketamine as adjuvant for acute pain management. Anesth Pain Med. 2019;9(6). e100178. doi: 10.5812/aapm.100178. [PubMed: 32280623]. [PubMed Central: PMC7119219]. 